\title{
Beta Cell Expression of Endogenous Xenotropic Retrovirus Distinguishes Diabetes-susceptible NOD/Lt from Resistant NON/Lt Mice
}

H. Rex Gaskins, Michal Prochazka, Kazuyuki Hamaguchi, David V. Serreze, and Edward H. Leiter

The Jackson Laboratory, Bar Harbor, Maine 04609

\section{Abstract}

Endogeneous retroviral expression in $\beta$ cells is a feature of prediabetes in nonobese diabetic (NOD) mice. The purpose of this study was to characterize the class-specific pattern of retroviral gene expression in NOD/Lt $\beta$ cells versus a related, but diabetes-resistant strain, NON/Lt. Electron microscopic comparison of $\beta$ cells from both strains indicated low constitutive expression of the intracisternal type A (IAP) retroviral class. However, NOD $\beta$ cells, in contrast to NON $\beta$ cells, expressed an additional intracisternal retroviral form resembling a type $C$ particle. Antibodies against both IAP and type $C$ were detected in NOD, with the humoral response to type $C$, but not IAP, preceding decline in $\beta$ cell function. RNA was extracted from freshly isolated islets from NOD and NON males. Comparative Northern blot analysis of total type $\mathrm{C}$ retroviral gene expression using a gag-pol DNA probe corroborated expression of endogenous type C proviruses in both NOD and NON islet cells and thymus. Use of class-specific retroviral probes identified the class of expressed endogenous retrovirus distinguishing the two inbred strains. The single ecotropic provirus present in both the NOD and NON genome $(E m v-30)$ was not expressed in islets or thymus of either strain. Comparison of endogenous xenotropic provirus content by Southern blot analysis revealed two unique xenotropic loci $(X m v-65,-66)$ in NOD; 8.4 and $3.0 \mathrm{~kb}$ xenotropic envelope (env) RNA transcripts were detected in NOD, but not NON islets and thymus. NON contained three xenotropic loci common to other inbred strains (Xmv-21, -25, and -28). Both strains were partially characterized for content of recombinant (polytropic and modified polytropic) proviruses. IAP RNA expression was common to both NOD and NON islets and hence could not be specifically associated with the unique intracisternal type $C$ particle found in NOD, but not NON $\beta$ cells. In conclusion, this study shows that expression of xenotropic type $C$ but not IAP distinguishes retroviral activity in NOD/Lt versus NON/Lt $\beta$ cells. The potential pathogenic role of retroviral gene expression in NOD $\beta$ cells is discussed. ( $J$. Clin. Invest. 1992. 90:2220-2227.) Key words: mice $\bullet$ nonobese diabetic • diabetes $\bullet$ endogenous retrovirus. $\bullet \beta$ cell

Address correspondence to Dr. Edward H. Leiter, The Jackson Laboratory, Bar Harbor, ME 04609. Dr. Gaskins's current address is University of Illinois, Urbana, IL. Dr. Prochazka's current address is National Institutes of Health, National Institute of Diabetes and Digestive and Kidney Diseases, Phoenix, AZ. Dr. Hamaguchi's current address is First Department of Medicine, Medical College of Oita, Oita, Japan. 1992.

Received for publication 7 January 1992 and in revised form 5 June

J. Clin. Invest.

(c) The American Society for Clinical Investigation, Inc.

$0021-9738 / 92 / 12 / 2220 / 08 \quad \$ 2.00$

Volume 90, December 1992, 2220-2227

\section{Introduction}

Insulin-dependent diabetes mellitus (IDDM) ${ }^{1}$ in the nonobese diabetic (NOD) mouse is an autoimmune disease whereby $\mathrm{T}$ lymphocytes selectively destroy the insulin-producing $\beta$ cells in pancreatic islets (1). An unresolved question is whether NOD $\beta$ cells express unique or strain-specific (neo)antigens required to target the initial autoimmune assault. Transplantation studies have indicated that diabetogenic genes in NOD do not encode a unique $\beta$ cell autoantigen, but instead create a dysregulated immune system producing $\mathrm{T}$ cells reactive against common $\beta$ cell products. A major component of NOD strain-specific diabetes susceptibility resides within bone marrow-derived cells. Diabetes resistance in F1 hybrids between NOD and diabetes-resistant strains (NON/Lt, CBA/J, $\mathrm{C} 57 \mathrm{BL} / 10 \mathrm{~J}$ ) can be abrogated by lethal irradiation and reconstitution with NOD bone marrow (2-5). After development of hyperglycemia, the NOD marrow-derived T cells autoreactive against $\mathrm{F} 1$ islets in situ rapidly rejected transplanted $\mathrm{NON} / \mathrm{Lt}$, $\mathrm{CBA} / \mathrm{J}$, or C57BL/10J islets respectively (3-5). The finding that NOD-derived CD-4 ${ }^{+} \mathrm{T}$ cell lines mediate islet damage in a non-MHC restricted fashion (6) provides further evidence that $T$ cells from diabetic mice respond to a cell product or products common to all mouse $\beta$ cells rather than to a $\beta$ cell autoantigen unique to NOD. However, in the transplantation studies, destruction of endogenous $\beta$ cells was initiated in situ before the demonstration of non-MHC restricted rejection of allogeneic islets engrafted into ectopic sites. Moreover, the $\mathrm{T}$ cell lines were derived from diabetic NOD donors and thus may have been exposed to $\beta$ cell antigens. Thus, the effectors that eliminate $\beta$ cells in a non-genotype-specific fashion may differ from those responsible for the initiation of necrosis in the islets in situ. Hence, a requirement for a NOD-specific pattern of gene expression at the $\beta$ cell level has not been formally excluded in the initiation of pathogenesis. For example, attraction of macrophages to NOD islets is considered to be an early pathogenic event in the process of insulitis development $(7,8)$. Although macrophages are normally not cytotoxic to nontransformed cells, they will attack neoplastic or virus-infected cells, raising the interesting possibility that NOD $\beta$ cells are expressing endogenous viral neoantigens.

An unusual morphologic feature characterizing the $\beta$ cell of the prediabetic NOD pancreas is the presence of an aberrant retrovirus budding into the cisternae of the rough endoplasmic reticulum (RER) $(9,10)$. The diameter and ultrastructure of

1. Abbreviations used in this paper: Emv, ecotropic murine leukemia virus; IAP, intracisternal type A particle; IDDM, insulin-dependent diabetes mellitus; MCF, mink cell focus-forming; $M p m v$, modified polytropic; NOD, nonobese diabetic; NON, diabetes-resistant NODrelated strain; $P m v$, polytropic murine leukemia virus; $r d$, retinal degeneration; RER, rough endoplasmic reticulum; scid, severe combined immunodeficiency; $X m v$, xenotropic murine leukemia virus. 
these particles suggested an immature type C particle (11); however, their intracisternal location within the RER has led others to classify these particles as another form of defective retrovirus, the intracisternal type A particle (IAP) (7). Interestingly, in CD-1, an ICR outbred strain related to NOD, morphologically identical retroviruses are induced in prenecrotic $\beta$ cells after multidose streptozotocin administration (12). Retroviral particles with the same type $C$ morphology as observed in NOD $\beta$ cells have not been observed in $\beta$ cells of NON/Lt, $\mathrm{CBA} / \mathrm{Lt}$, or C57BL strains, although IAP have been observed in NOD/Lt, NON/Lt, and CBA/J $(13,14)$. Since cyclophosphamide acceleration of insulitis and diabetes in prediabetic male NOD mice has been correlated with upregulated expression of these uncharacterized particles (7), it was important to unambiguously identify the type of retroviral genome being expressed (ecotropic or xenotropic type $\mathrm{C}$, or intracisternal type $\mathrm{A}$ ). In the present report we demonstrate that expression of the xenotropic type $\mathrm{C}$ retroviral class distinguishes NOD/Lt from NON/Lt $\beta$ cells. The strain-specific proviral content has also been partially characterized for both inbred strains to permit genetic analysis of the potential of endogenous type $C$ retroviruses to function as modifiers of diabetes susceptibility.

\section{Methods}

Mice. NOD/Lt and NON/Lt inbred mice were maintained by brother-sister mating in our research colony at The Jackson Laboratory (Bar Harbor, ME). NOD/LtSz mice congenic for the severe combined immunodeficiency ( scid) mutation were kindly provided by Dr. L. D. Shultz of this institution. All mice were maintained in a specific pathogen-free facility on a 14:10 light/dark cycle and were allowed free access to diet 96W (Emory Morse Co., Guilford, CT) and chlorinated drinking water. Diabetes incidence of NOD/Lt is $83 \%$ in females and $43 \%$ in males at 42 wk of age. NON/Lt are diabetes resistant (15).

Diabetes monitoring. Development of diabetes was monitored monthly by screening for glycosuria with Tes-Tape (kindly provided by Eli Lilly and Co., Indianapolis, IN). Glycosuria of $>2 \%$ at two consecutive tests was considered diagnostic for diabetes.

Electron microscopy. Pancreatic islets obtained for ultrastructural observation were from 5- to 7-wk-old NOD/Lt female mice. Dissected pancreases were fixed in $2 \%$ glutaraldehyde, $1 \%$ paraformaldehyde in $0.1 \mathrm{M}$ sodium cacodylate ( $\mathrm{pH} 7.2$ ). After fixation, islets were hand microdissected with the aid of a dissecting microscope (Wild Heerbrugg Instruments Inc., Farmingdale, NY), postfixed in $1 \% \mathrm{OsO}_{4}$, and embedded in paraffin. Ultrathin sections were examined with a transmission electron microscope (JEOL, USA, Peabody, MA).

Autoantibody determinations. Development of autoantibodies against $\mathrm{p} 73$, the IAP group-specific antigen and whole reticuloendothelial virus (REV), a type $\mathrm{C}$ retrovirus, in aging NOD/Lt female mice were determined by ELISA as described previously (16). These reagents were respectively provided by Drs. E. L. Kuff and S. Oroszlan, (National Cancer Institute). The preparation of complete reticuloendothelial virus virions shares many antigenic determinants with murine type $C$ retroviruses (17) such that any antibodies binding this preparation would be elicited by endogenous type $C$ determinants. Pancreatic insulin content per milligram of protein was determined for a separate group of aging females. At killing, the pancreases were bisected longitudinally and half was extracted in acid ethanol as described previously (18). Insulin in the extract was determined by radioimmunoassay, with content expressed as micrograms per milligram extract protein.

Recombinant DNA probes. Recombinant DNA probes for the ecotropic $(E m v)$ proviral envelope (env) sequence (clone pEco; [19]); xenotropic $(X m v) e n v$-specific (NZB-IU-6), a 100-bp BstNI-XmaI subclone of the mink cell focus-forming (MCF) envelope gene (20); and chicken beta-actin were provided by Dr. H. Bedigian (The Jackson Laboratory). A 4.6-kb insert of AKR MuLV clone 623 was used to detect total type $C$ transcripts (21). Oligonucleotide probes (JS-6, JS10 ) were also used to detect $X m v$-specific sequences (22) and were synthesized on a Applied Biosystems DNA synthesizer (Applied Biosystems, Inc., Foster City, CA). A mouse preproinsulin I cDNA probe was provided by Dr. A. Permutt (Washington University, St. Louis, $M O)$. Purified cDNA inserts were labeled with [ $\left.{ }^{32} \mathrm{P}\right] \mathrm{dCTP}$ by the random hexamer-primer procedure (23) with an oligonucleotide-labeling kit (Pharmacia Inc., Piscataway, NJ). Oligonucleotide probes were labeled as described previously (24).

Northern blot analysis. A single-step guanidinium thiocyanate method (25) was used to isolate total cellular RNA from freshly isolated islets from 5- to 6-wk-old NOD/Lt and NON/Lt males, cloned islet cell lines, and pooled thymi from 16-d-old fetal and 7-d-old postnatal NOD/Lt and NON/Lt female mice. Northern blot analysis ( 20 $\mu \mathrm{g}$ per lane) by electrophoresis in a $1.25 \%$ agarose gel, transfer to nylon membrane filters (Zetabind; Cuno Laboratory Products, Meriden, CT), and hybridization were performed as described previously (26). The equivalence in quantity and quality of RNA loaded in each lane of a gel was determined by an ultraviolet-shadowing technique (27) before hybridization. Transcript size was determined by comparing migration distance with a RNA ladder and 28S and 18S RNA.

Strain comparison of endogenous proviruses. Ecotropic (Emv), xenotropic $(X m v)$, polytropic $(P m v)$, and modified polytropic $(M p m v)$ proviral loci were identified by restriction fragment length variant analysis as previously described $(24,28,29)$. For Southern blot analysis, high molecular weight DNA was prepared from fresh or frozen tissues by a modification of the method described in (30). After two phenol/chloroform extractions, the DNA was precipitated with ethanol. For Southern blot analysis, DNA ( $10 \mu \mathrm{g}$ per lane) was digested overnight with indicated restriction endonuclease under conditions recommended by the manufacturer (Gibco BRL, Gaithersburg, MD). Enzyme-restricted DNA was separated by electrophoresis in $0.7 \%$ agarose gels, transferred to nylon membrane filters (Zetabind; CUNO) in $10 \times \operatorname{SSC}(1 \times \operatorname{SSC}=0.15 \mathrm{M} \mathrm{NaCl}, 0.015 \mathrm{M} \mathrm{Na}$ citrate $)$, and crosslinked to the filters using a UV-Stratalinker (Stratagene Inc., LaJolla, CA). Hybridization conditions and autoradiography were as described previously (31). The final two posthybridization washes were performed at $65^{\circ} \mathrm{C}$ in $0.1 \times$ SSC- $0.1 \%$ SDS for $30 \mathrm{~min}$ each, or at $62^{\circ} \mathrm{C}$ in $2 \times$ SSC $-0.2 \%$ SDS for 15 min each when using the JS- 6 and JS- 10 oligonucleotide probes (24).

\section{Results}

Ultrastructural analysis of retroviruses in pancreatic $\beta$ cells of $N O D / L t$ mice. Within NOD/Lt islets, retroviral particles were observed only in insulin-producing $\beta$ cells. Two morphologically distinct types of retroviral particles were observed within NOD/Lt $\beta$ cells, clearly identifiable IAP and another morphologic type differing from IAP by the presence of an additional unit membrane structure in the envelope (Fig. $1 A$ ). This latter morphology was identical to that identified as type $C$ in $\beta$ cells of CD-1 mice after streptozotocin treatment (12). In cross-sectional profile, these latter particles often appeared as the elongated tubular forms (Fig. $1 B$ ) described by others $(7,9,10)$. Both IAP and the apparent type $C$ particles were exclusively found within the cisternae of the RER. Mature type $C$ particles were not observed at the cell surface of NOD/Lt $\beta$ cells, indicating the defective nature of the putative type $\mathrm{C}$ genome being expressed. In $\beta$ cells from both prediabetic NOD/Lt and NON/Lt mice, constitutive IAP expression at low levels was demonstrable ultrastructurally. IAP were observed in $\sim 10 \%$ of NOD $\beta$ cells surveyed per islet with the greatest density being found in partially degranulated $\beta$ cells. The density of type $C$ particles was less than that of IAPs with no obvious relation to 

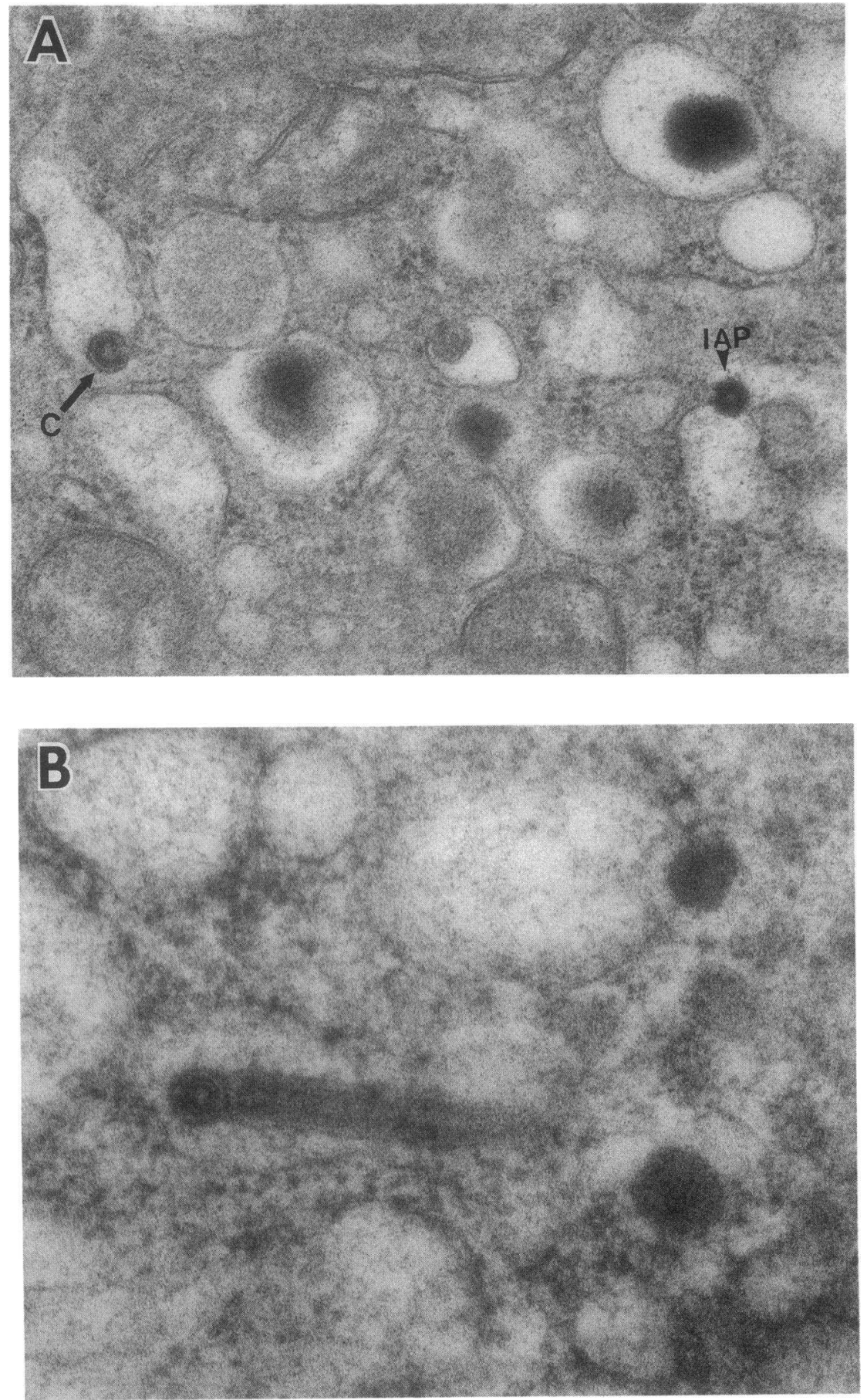

Figure 1. Ultrastructural appearance of intracisternal retroviruses present in a $\beta$ cell from a prediabetic 10-wk-old NOD/Lt female. $(A)$ An intracisternal type A particle (IAP) is distinguishable from an intracellular type $C$ retrovirus $(C)$ by the extra unit membrane (trilaminar viral nucleocapsid) surrounding the latter $(139,000 \times)$. (B) Cross-sectional view of an intracisternal type $C$ particle in a $\beta$ cell revealing a elongated cylindrical structure $(260,000 \times)$. the degree of $\beta$ cell granulation. In NOD/Lt islets it was not uncommon to observe IAP and type $\mathrm{C}$ retroviral particles within the same $\beta$ cell as depicted in Fig. $1 A$. Ultrastructural survey of NON/Lt islets revealed the presence of IAP, but not the putative type $C$ particles, in $\beta$ cells.

Retroviral autoantibody development in NOD/Lt mice. Serum samples were obtained from NOD/Lt female mice bled sequentually at 6,10 , and $18 \mathrm{wk}$ of age to determine the time course of autoantibody responses to IAP and type $\mathrm{C}$ retroviral antigens. The data are presented in relation to shifts in pancreatic insulin content that accompanies the prediabetic state (Fig. 2). Mean pancreatic insulin content was normal until 12 wk of age, with an abrupt decline occurring between weeks 12 and 14 (Fig. 2). Autoantibody titers to type $\mathrm{C}$ and IAP retroviral antigens were low $(<1 / 50)$ at 6 wk of age. By 10 wk of age, before the decline in mean insulin content (and development of hyperglycemia), antitype $\mathrm{C}$ autoantibody titers had increased $(\sim 1 / 100)$, whereas autoantibody titers to IAP antigens remained low. Overt hyperglycemia was first observed at $14 \mathrm{wk}$ of age in these animals. By 18 wk of age, when females were becoming overtly diabetic (data not shown), significant autoantibody titers to both type $C$ and IAP retroviral antigens were present. Thus, autoantibodies to type $C$ antigens preceded widespread $\beta$ cell necrosis whereas autoantibodies to IAP antigens developed concomitant with the development of hyperglycemia. 


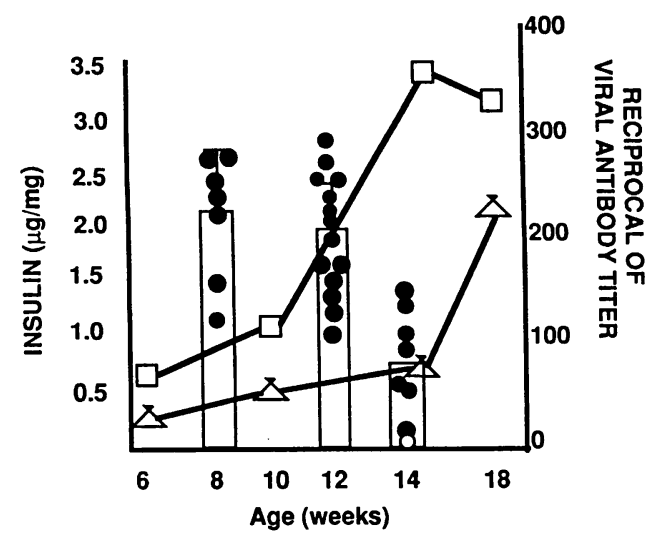

Figure 2. Rise in autoantibody titer against type $C(\square)$, but not IAP $(\Delta)$ coincides with activation of $\beta$ cell necrosis as assessed by decline in pancreatic insulin content. Antibody titers plotted represent the average titer of five to eight serum samples per age group. The bars represent the average insulin content for groups of normoglycemic females whose individual insulin contents are shown by the closed circles. One female overtly hyperglycemic at $14 \mathrm{wk}$ is shown by the open circle. Diabetes incidence in this sample population was $80 \%$ by $40 \mathrm{wk}$.

Retroviral gene expression in NOD/Lt and NON/Lt islets. Type $C$ retroviral subclass specificity is based on host range determinants (ecotropic, replicating only in mouse cells and encoded by $E m v$ proviral loci; xenotropic, replicating in cells of heterologous species and encoded by $X m v$ proviral loci). In addition, another class (MCF), when infectious, represents recombinants between ecotropic and nonecotropic viruses and can be subdivided into polytropic $(P m v)$ and modified polytropic $(M p m v)$ subclasses. Although gag-pol gene sequences are generally conserved among the type $C$ subclasses, sequence differences in the env genes permit differentiation of $E m v$-versus $X m v$ - and MCF-encoded transcripts. Comparative Northern blot analysis of total type $\mathrm{C}$ retroviral gene expression using a gag-pol recombinant DNA probe is shown in Fig. $3 \mathrm{~A}$. Transcripts of 10 - and 8.4-kb encoding gag-pol gene products were highly expressed in NOD/Lt islets whereas only a low level of a 10-kb RNA transcript was observed in NON/Lt islets. Hybridization with type $\mathrm{C}$ env-specific probes revealed that neither NOD/Lt nor NON/Lt islets were expressing Emv-specific env transcripts (not shown). However, NOD/Lt islets but not NON/Lt islets were expressing 8.4- and 3.0-kb $X m v$-specific env RNA transcripts (Fig. $3 B$ ). Since for all type $\mathrm{C}$ retroviral classes gag-pol is spliced from the complete $8.4-\mathrm{kb}$ transcript, the gag-pol specific probe does not hybridize with the $3.0-\mathrm{kb}$ transcript recognized by the Xmv-env-specific probe. MCF mRNA transcripts were not detected in either NOD/Lt or $\mathrm{NON} / \mathrm{Lt}$ islets. NOD/Lt and NON/Lt islets expressed similar levels of insulin RNA (Fig. $3 C$ ). AKR/J thymus and the mouse $\mathrm{C} 1300$ neuroblastoma cell line, both of which express high levels of type C RNA, were included as controls for Northern blot analysis. Equivalence of RNA loaded per lane was demonstrated by the similar levels of actin RNA in each preparation (Fig. $3 \mathrm{D}$ ).

Retroviral gene expression in NOD/Lt and NON/Lt thymus. Similar levels of total type $C$ retroviral gene expression detected as 10- and 8.4-kb RNA transcripts after hybridization with a gag-pol cDNA probe were found in NOD/Lt and NON/ Lt thymus. Likewise, MCF env-specific RNA transcripts were

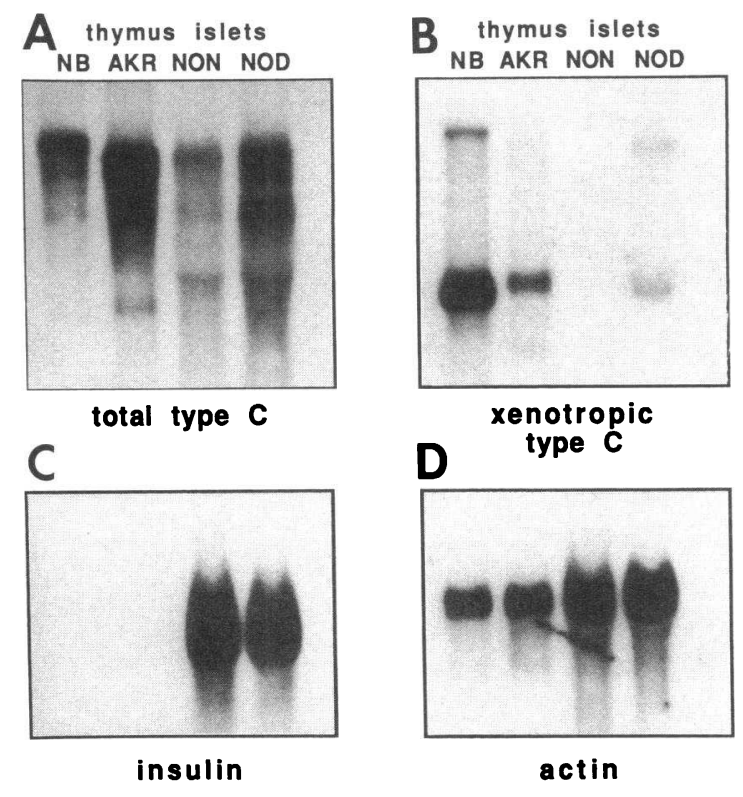

Figure 3. Northern blot analysis of total and xenotropic-specific type $\mathrm{C}$ retroviral gene expression in NOD/Lt and NON/Lt islets. $20 \mu \mathrm{g}$ of total RNA from freshly isolated NOD/Lt and NON/Lt islets, $\mathrm{AKR} / \mathrm{J}$ thymus, and $\mathrm{C} 1300$ neuroblastoma $(N B)$ cells (the latter two samples comprised positive controls) was size separated by electrophoresis in a $1.25 \%$ agarose gel and transferred to a nylon membrane. The RNA blot was then hybridized sequentially with the following $\left[{ }^{32}\right.$ P]labeled DNA probes: $(A)$ for total type $C$ expression, a nonclass-specific type $\mathrm{C}$ gag-pol probe (8.4- and 5.6-kb major transcripts), $(B)$ a xenotropic class-specific envelope probe (8.4- and 3.0$\mathrm{kb}$ transcripts), $(C)$ preproinsulin, and $(D)$ actin. Transcript size was determined by comparing migration distance with a RNA ladder and $28 \mathrm{~S}$ and 18S RNA. A 13-d autoradiograph is depicted. Results are representative of two independent analyses.

detected in both NOD/Lt and NON/Lt thymus and Emvenvspecific transcripts were not detected in thymus from either strain. As for islets, Xmv env transcripts were present in NOD/ $\mathrm{Lt}$ thymus but not in NON/Lt thymus. Longitudinal analysis revealed significant levels of total type $\mathrm{C}$ transcripts in fetal thymus of both strains by day 16 (Fig. $4 A$ ). The strain-specific pattern of $X m v$ env expression was also established at this fetal age (Fig. $4 \mathrm{~B}$ ). Postnatal age did not significantly affect the level of total type C RNA expression or the NOD-specific expression of $X m v$ env RNA as similar results were obtained using thymuses from 16-d fetal, and 7-d and 7-wk postnatal $\mathrm{NOD} / \mathrm{Lt}$ and NON/Lt mice. Although devoid of mature thymocytes (32), thymuses from NOD-scid/scid mice expressed levels of total type $\mathrm{C}$ and $X m v$-specific RNA transcripts similar to that of NOD/Lt mice (Fig. 4). Hence, stromal elements rather than thymocytes may be the site of xenotropic retrovirus expression in this tissue.

Identification of endogenous proviruses in NOD/Lt and $N O N / L t$. The number of endogenous $X m v$ proviruses in $\mathrm{NOD} / \mathrm{Lt}$ and NON/Lt was determined by Southern blot analysis using the $X m v$-specific oligon acleotide env probe JS-6/JS10 as described in Methods. In EcoRI- or PvulI-digested mouse genomic DNA, this probe hybridizes to 3' proviral DNA-cellular DNA junction fragments each corresponding to a different $X m v$ locus (33). Because the EcoRI and PvuII sites marking and distinguishing individual proviruses are well conserved, 

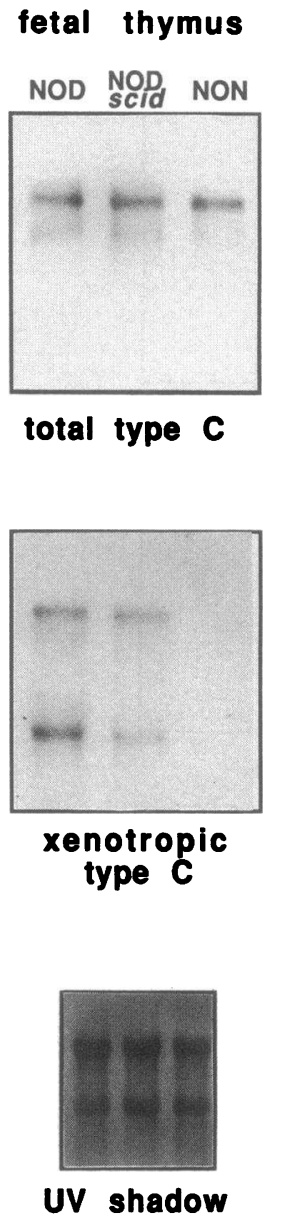

Figure 4. Northern blot analysis of total type $\mathrm{C}$ and xenotropic type $\mathrm{C}$ retroviral gene expression in NOD/Lt and NON/Lt thymus. $20 \mu \mathrm{g}$ of total RNA from 16-d fetal NOD/Lt, NOD/LtSz-scid/scid, and NON/Lt thymus was analyzed as described for Fig. 3. The blot was hybridized sequentially with nonclass-specific type C (gag-pol) and xenotropic env-specific ${ }^{32} \mathrm{P}$-labeled DNA probes. Transcript sizes were the same as described in the legend to Fig. 3. An ultraviolet shadow of the blot is shown in the bottom panel to demonstrate that equivalent amounts of RNA are present in each lane.

specific fragment sizes generated by both restriction endonucleases are diagnostic for each $X m v$ locus $(28,33)$.

Analysis of kidney DNA digested with EcoRI and PvuII revealed the presence of three $X m v$ loci in NOD/Lt and of four $X m v$ loci in NON/Lt (Fig. $5 A$, and Table I). The only fragment shared between both strains and characterized by a weak hybridization signal was identified as $X m v-6$, known to be a $X m v$-related, incomplete proviral copy present in the genome of all mouse strains examined (33). The two additional $X m v$ elements in NOD/Lt detected as 3.8- and 7.5-kb EcoRI fragments (Fig. $5 \mathrm{~A}$ ) were different from all published $X m v$ loci (33) and were given symbols $X m v-65$ and $X m v$-66, respectively (Fig. $5 \mathrm{~A}$ ). Comparison of EcoRI and PvulI fragment patterns of NON/Lt with other inbred strains identified the three NONcharacteristic proviruses as $X m v-21(\mathrm{Chr} 1), X m v-25(\mathrm{Chr} 9)$, and $X m v-28$ (Chr 5) (Fig. $5 A$ and Table I). $X m v-25$ is closely-

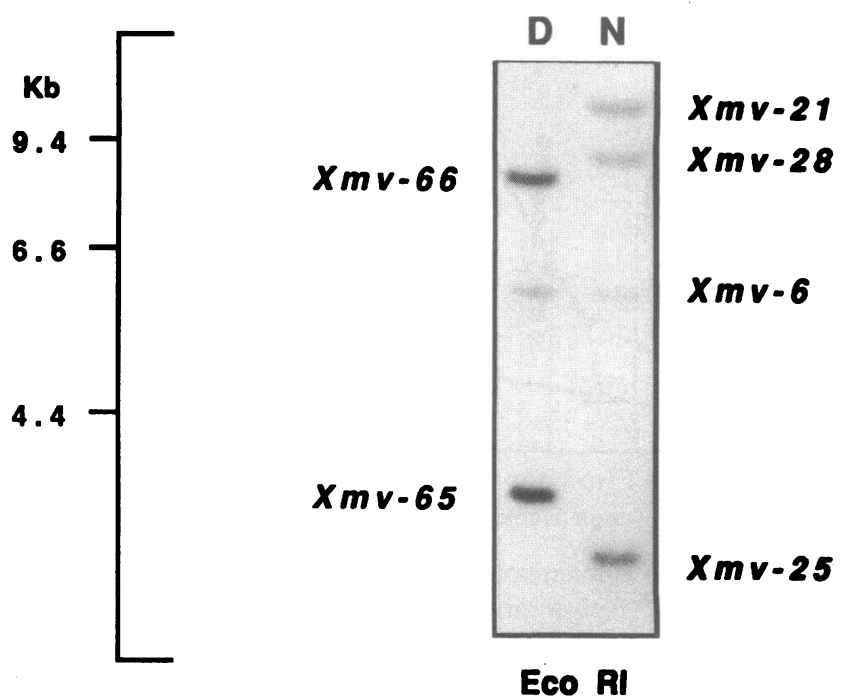

Figure 5. Restriction fragment length variant analysis demonstrating $X m v$ differences between NOD/Lt $(D)$ and NON/Lt $(N)$ mice. Southern blots of EcoRI-digested DNA were hybridized with ${ }^{32} \mathrm{P}$-labeled xenotropic- $(\mathrm{Xmv}$-) specific envelope probe JS-6/JS-10 as described in Methods. Known chromosomal locations are provided in Table I. The scale at the left side indicates sizes in kb of HindIII-digested $\lambda$ phage DNA fragments.

linked to $T h y-1$ and genes of the $T 3 d$ complex, a chromosomal region associated with incompletely dominant diabetes resistance in outcross of NOD/Lt with NON/Lt (31). It has been reported that $X m v-28$ is closely linked to, and possibly causally associated with, the recessive mutation retinal degeneration $(r d)$ on Chr 5 in inbred strains suffering from this eye disorder (33). Eye examination of NON/Lt mice revealed phenotypic ocular abnormalities diagnostic for retinal degeneration demonstrating that this $X m v$-28-positive strain also carries the $r d$ mutation. No eye abnormalities were seen in NOD/Lt (Roderick, Dr. T., The Jackson Laboratory, personal communication ). Genetic segregation analysis has not yet indicated a chromosomal location for $X m v-65$. Provisional assignment of $X m v-$ 65 to $\mathrm{Chr} 17$ has been made since, in contrast to standard NON/Lt mice, this provirus is present in an NON/Lt stock congenic for the MHC $\left(H-2^{g 7}\right)$ from NOD/Lt.

As shown in Table I, the NOD/Lt genome contains the previously mapped $P m v-8,-14$, and -41 , as well as $M p m v-4,-6$, $-7,-9$, and -18 proviral loci. The NON/Lt genome contains the previously mapped $P m v-3,-19$, and -33 , as well as $M p m v-1$ and -13 proviral loci. In addition, these two strains contain additional Pmv and $M p m v$ proviral loci that are unique to NOD

Table I. Comparison of Endogenous Ecotropic, Xenotropic, Polytropic, and Modified Polytropic Proviral Loci in NOD/Lt and NON/Lt Mice*

\begin{tabular}{|c|c|c|c|c|c|c|c|c|c|c|c|c|c|c|c|c|c|c|c|}
\hline & $\begin{array}{c}E 30 \\
11\end{array}$ & $\begin{array}{c}X 21 \\
1\end{array}$ & $\begin{array}{c}X 25 \\
9\end{array}$ & $\begin{array}{c}X 28 \\
5\end{array}$ & $\begin{array}{c}X 65 \\
?\end{array}$ & $\begin{array}{r}X 66 \\
17^{\ddagger}\end{array}$ & $\begin{array}{l}P 3 \\
12\end{array}$ & $\begin{array}{l}P 8 \\
10\end{array}$ & $\begin{array}{c}P 14 \\
16\end{array}$ & $\begin{array}{c}\text { P19 } \\
4\end{array}$ & $\begin{array}{c}P 33 \\
2\end{array}$ & $\begin{array}{c}P 41 \\
13\end{array}$ & $\begin{array}{c}M 1 \\
7\end{array}$ & $\begin{array}{c}M 4 \\
11\end{array}$ & $\begin{array}{c}M 6 \\
1\end{array}$ & $\begin{array}{c}M 7 \\
5\end{array}$ & $\begin{array}{c}M 9 \\
3\end{array}$ & $\begin{array}{c}M 13 \\
5\end{array}$ & $\begin{array}{c}M 18 \\
11\end{array}$ \\
\hline $\mathrm{NOD} / \mathrm{Lt}$ & + & - & - & - & + & + & - & + & + & - & - & + & - & + & + & + & + & - & + \\
\hline $\mathrm{NON} / \mathrm{Lt}$ & + & + & + & + & - & - & + & - & - & + & + & - & + & - & - & - & - & + & - \\
\hline
\end{tabular}

* Provisional assignment to previously-defined proviral loci determined as previously described (24, 29, 32-33). E, Emv; X, Xmv; P,Pmv; $M$, $M p m v .{ }^{\ddagger}$ Provisional localization based upon presence of this locus in the NON.NOD- $H-2^{27}$ congenic strain. 
and/or NON, but are absent in other inbred strains (Frankel, Wayne, The Jackson Laboratory, personal communication).

\section{Discussion}

The purpose of this investigation was to identify the retroviral subclass encoding the aberrantly budding intracisternal particle present in $\beta$ cells of NOD/Lt, but not the related NON/Lt strain. Candidate loci include IAP, ecotropic, xenotropic, or mink cell focus forming ( $\mathrm{MCF}$ ) type $\mathrm{C}$ proviruses. The present results clearly demonstrate that NOD/Lt islets can be distinguished from NON/Lt islets by expression of endogenous xenotropic proviral gene(s). These transcriptional differences were reflected at the cellular level by the presence of defective intracellular budding type C-like particles in NOD/Lt, but not NON/Lt $\beta$ cells. Although similar levels of total type C gene expression were detected in NOD/Lt and NON/Lt islets, xenotropic transcripts were unique to NOD/Lt $\beta$ cells, correlating with the ultrastructural presence of the defective intracellular type $C$ retroviral particle. That the aberrant type $C$ particle present in NOD/Lt $\beta$ cells is of xenotropic origin is further supported by the apparent absence of MCF mRNA transcripts in these cells. Given that the two strains are distinguished by differences in xenotropic, but not total type $\mathrm{C}$ expression, the defective particles expressed in NOD $\beta$ cells are most likely encoded by $X m v-65$ and/or $X m v-66$, since these are the only xenotropic proviral loci present in NOD/Lt but absent in NON/Lt. In contrast, IAP transcripts and ultrastructurally identifiable IAP were present in islets of both strains. Thus, the present results indicate that, despite their intracisternal location, the retroviral particles distinguishing NOD from NON $\beta$ cells are not encoded by an IAP proviral locus (or loci) as was suggested by Suenaga and Yoon (7), but rather present a defective xenotropic type C particle. Neither NOD/Lt nor NON/Lt islets express $E m v-30$, the single ecotropic proviral gene on $\mathrm{Chr}$ 11 present in these strains (34), whereas both express MCF RNA transcripts. Proof that the aberrant tubular forms of intracisternal retrovirus observed in NOD $\beta$ cells in situ is not of ecotropic origin is provided by NOR/Lt mice, a recently constructed inbred strain containing $X m v-65$ and $X m v-66$, but lacking the $E m v$-30 locus (29). As in NOD/Lt mice, ecotropicnegative NOR/Lt mice express aberrant tubular forms of intracisternal retroviruses in $\beta$ cells.

Support for the intracellular type $C$ particles being encoded by xenotropic and not ecotropic type C or IAP genes is provided by our previous analysis of class-specific endogenous retroviral expression in the transformed NOD-derived $\beta$ cell line, NIT-1, (35). NIT-1 cells do not produce the defective intracellular $C$ type particles observed in NOD $\beta$ cells in situ, and we have been unable to detect $X m v$ env gene transcripts in these cells (data not presented). Transformed NIT-1 cells differed from standard NOD/Lt $\beta$ cells in that they expressed MCF mRNA transcripts (data not shown). Furthermore, NIT-1 cells expressed high levels of infectious ecotropic type $\mathrm{C}$ particles (35). As discussed above, expression of the single ecotropic locus $(E m v-30)$ is repressed in standard NOD/Lt mice. These alterations in retroviral expression in NIT-1 cells presumably are a consequence of neoplastic transformation since Emv-30 was also expressed at high levels in NOD-scid/scid thymomas (32). However, IAP expression is common to both SV40transformed NIT-1 cells and nontransformed NOD $\beta$ cells. $(26,35)$. Thus, whereas IAP and ecotropic type $C$ particles were both expressed in the absence of xenotropic env gene tran- scripts, the tubular intracisternal retroviral particles differentiating NOD/Lt from NON/Lt $\beta$ cells and from NIT- 1 cells correlated with the presence of xenotropic env gene transcripts. In addition, $\mathrm{p} 30$, the group-specific antigen of type $\mathrm{C}$ retroviruses, has recently been identified by Western blot in lysates from NOD islets (36).

Intracisternal forms of type $\mathrm{C}$ retroviral particles have been associated with cytopathology of several disease phenotypes in mice (37-41), including those observed in prenecrotic CD-1 $\beta$ cells after multidose streptozotocin administration (12). Moreover, a correlation between the initiation of severe diabetes and induction of these intracellular retroviral particles in cyclophosphamide-induced diabetes in NOD male mice has recently been reported by Suenaga and Yoon (7). These latter authors concluded that the particles were exclusively IAP on the basis of their intracisternal localization. IAP do not have an extracellular phase because the env gene is defective and functional envelope proteins are not translated. Sequestration of IAP within the RER occurs because the 5' end of the gag gene coding for the 73,000-D IAP core protein (p73) contains a hydrophobic $\mathrm{NH}_{2}$-terminus "signal" sequence that directs the core protein into the RER lumen (42). This hydrophobic leader sequence apparently is not excised by a serine protease and thus anchors the p73 to the inside face of the RER. "Budding" into the lumen of the RER occurs as a result of infolding of the inner unit membrane of the RER around the $p 73$ core protein, two strands of virion RNA, and the polymerase. The possibility cannot be excluded that the intracisternal tubular forms with an additional unit membrane not characteristic of IAP may be a hybrid product of a genetic recombination between an IAP and a xenotropic gene. Indeed, a new class of mouse genomic elements designated intracisternal A-particlerelated elements coding for envelope has recently been identified in which the env gene is distinct from a standard (defective) IAP gene and is potentially expressible (43). Technical limitations have thus far prevented isolation of sufficient quantities of these tubular particles from NOD islets to permit molecular analysis. The particles are replication defective since homogenates of NOD islets with appropriate ecotropic and xenotropic target cell lines did not yield infectious virus.

Immunochemical staining of islets from prediabetic 10-wkold NOD mice at the light microscopic level with a rabbit antiserum against xenotropic Kirsten murine sarcoma virus (NIHxeno) indicated that only a subset of islet cells contained detectable levels of the antigen (10). The frequency at which the type C-like intracisternal particles was observed by electron microscopy in that study (10) as well as the present one confirmed that expression was limited to a subset of the $\beta$ cells. Also supporting the low frequency of the defective xenotropic particle is the relatively low hybridization signal obtained with $X m v$-env-specific probes in Northern blot analysis (Fig. $3 \mathrm{~B}$ ). The present observations as well as all previous reports of type $C$ particles in NOD islets demonstrate that expression is limited to $\beta$ cells and not observed in $\alpha, \delta$, and PP cells. It has not yet been established whether both, or only one, of the $X m v$ loci are expressed in NOD/Lt $\beta$ cells. The unique $H-2^{g 7}$ haplotype of NOD on Chr 17 contributes a major component of genetic susceptibility to IDDM (15). Preliminary genetic mapping of $X m v-66$ indicates it is located on $\mathrm{Chr} 17$ between $\mathrm{Tu}-66$ and $H-2 K$. Hence, $\beta$ cell-specific expression of $X m v-66$ could represent an additional component of the $\mathrm{Chr} 17$-associated diabetes susceptibility. 
Numerous mechanisms whereby endogenous retroviral expression in pancreatic $\beta$ cells could lead to diabetes could be proposed and include the obvious possibility that retroviral proteins contribute to a spectrum of antigens capable of triggering $\beta$ cell autoimmunity in NOD mice. Type $C$ retroviral products exhibit a high degree of homology with MHC class I determinants (44). Recently we have identified an MHC class I cross-reactive determinant inducible by interferon-gamma that is expressed in NOD macrophages and $\beta$ cells, to which primed NOD T cells respond (45). Acceleration of diabetes development in NOD mice by cyclophosphamide treatment has been associated with increased $\beta$ cell retroviral content (7). In the present study, the observation that antibody titers to type $C$ antigens increased relatively early in the prediabetic period and reached a peak when widespread $\beta$ cell necrosis was initiating may in part reflect $\mathrm{T}$ cell responses to retroviral determinants being presented by antigen-presenting cells in the insulitic infiltrates. Since the early insulitic infiltrates contain a polyclonal population of $T$ cells (46), $T$ cell responses to retroviral antigens undoubtedly reflect only a portion of the total immune response. It cannot be excluded that the different ontogenies of antibody development against type $\mathrm{C}$ and IAP antigens represent epiphenomena that are secondary to earlier occurring primary pathogenic events.

Regardless of whether endogenous retroviral expression represents an important mechanism in initiation of $\mathrm{T}$ cell-mediated autoimmune reactions against $\beta$ cells, intracellular accumulation of retroviral proteins may comprise $\beta$ cell function by interfering with regulated secretory pathways or otherwise perturb intracellular trafficking. IAPs have been proposed to play such a role in $\mathrm{C} 57 \mathrm{BL} / \mathrm{KsJ} \beta$ cells, making this strain more susceptible to the diabetogenic actions of the recessive $d b \mathrm{mu}$ tation than the closely related IAP-nonexpressing C57BL/6J strain (47). Expression of an endogenous type $C$ retroviral genome in $\mathrm{C} 3 \mathrm{H}-\mathrm{db} / \mathrm{db}$ mice was associated with accelerated pathogenesis in diabetes-susceptible males and abrogation of resistance in females (18). Development of diabetes in transgenic mice in which $\beta$ cells are hyperexpressing certain proteins confirms that the viability of this cell type can indeed be compromised by aberrant expression of intracellular proteins (48).

Other suggestions for involvement of endogenous retroviral gene products in mechanisms of autoimmunity come from studies by Krieg and coworkers $(49,50)$ in which they demonstrate that thymic-specific expression of $8.4 \mathrm{Mpm} v$ RNA transcripts is characteristic of several inbred mouse strains with a predisposition to murine lupus. More recently, these authors have demonstrated that the high level of constitutive thymic $M p m v$ expression in lupus-prone mice maps to a bone marrow-derived precursor cell (51). Interestingly, the abnormal tolerance characteristic of lupus-prone mice also is associated with defects in bone marrow-derived stem cells as is the abnormal accessory cell function characteristic of autoimmune diabetes in NOD mice $(2,3)$. The fact that MCF RNA expression was similar in NOD/Lt and NON/Lt thymus indicates that NOD mice do not share with murine lupus-prone mice the aberrant thymic pattern of $M p m v$ expression (50). However, the strain-specific pattern of $X m v$-env RNA expression in thymus described in the present study could underlie abnormal tolerance induction as proposed for aberrant $M p m v$ expression in lupus-prone strains.

Retroviral loci account for as much as $0.1-0.5 \%$ of the total mouse genome and a significant number of these endogenous proviral loci are found in linkage with genes that encode histocompatibility and lymphocyte differentiation antigens (52, 53). Furthermore, it has recently been demonstrated that endogenous $M t v$ retroviral sequences are homologous to $M l s$ (minor lymphocyte stimulatory) superantigens and thus serve to alter the $\mathrm{T}$ cell repertoire (54-57). These observations raise the interesting possibility that $X m v$ gene products in addition to representing $\beta$ cell autoantigens may also affect pathogenesis in NOD mice by altering the $\mathrm{T}$ cell selection process. The unique $H-2^{g 7}$ haplotype of NOD contributes a major component of genetic susceptibility to IDDM (15). If $\mathrm{H}-2^{g 7}$ linkage for $X m v-66$ is confirmed, and if $X m v-66$ expression contributes to the tissue-specific patterns described herein, then this xenotropic locus extends the diabetogenic haplotype. Whether retroviral expression, or lack thereof, underlies abnormal tolerance induction in the autoimmune-prone NOD mouse awaits more detailed characterization of the class specificity and ontogeny of retroviral gene expression in NOD bone marrow, thymus, and islets, as well as cells regulating peripheral tolerance.

\section{Acknowledgments}

Dr. Wayne Frankel is thanked for his assistance in identification of proviral loci. The skilled technical assistance of Ms. Sherri Worthen is gratefully acknowledged.

This work was supported by National Institutes of Health grants DK 36175 and DK 27722 and a postdoctoral fellowship from the Juvenile Diabetes Foundation, International (H. R. Gaskins).

\section{References}

1. Harada, M., and S. Makino. 1986. Immunological manipulation of diabetes production in NOD mice. In Insulitis and Type I Diabetes. Lessons from the NOD Mouse. S. Tarui, Y. Tochino, and K. Nonaka, editors. Academic Press, Tokyo. 143-153.

2. Serreze, D. V., and E. H. Leiter. 1991. Development of diabetogenic T cells from NOD/Lt marrow is blocked when an allo-H-2 haplotype is expressed on cells of hemopoietic origin, but not on thymic epithelium. J. Immunol. 147:1222-1229.

3. Serreze, D. V., E. H. Leiter, S. M. Worthen, and L. D. Shultz. 1988. NOD marrow stem cells adoptively transfer diabetes to resistant (NOD $\times$ NON) F1 mice. Diabetes. 37:252-255.

4. Serreze, D. V., and E. H. Leiter. 1990. Transplantation analysis of beta cell destruction in (NOD $\times$ CBA )F1 bone marrow chimeras. Diabetologia. 33:8492.

5. Wicker, L. S., B. J. Miller, A. Chai, M. Terada, and Y. Mullen. 1988 Expression of genetically determined diabetes and insulitis in the nonobese diabetic (NOD) mouse at the level of bone marrow-derived cells. Transfer of diabetes and insulitis to nondiabetic $($ NOD $\times \mathrm{B} 10) \mathrm{F} 1$ mice with bone marrow cells from NOD mice. J. Exp. Med. 167:1801-1810.

6. Haskins, K., M. Portas, B. Bergman, K. Lafferty, and B. Bradley. 1989. Pancreatic islet-specific T-cell clones from nonobese diabetic mice. Proc. Natl. Acad. Sci. USA. 86:8000-8004.

7. Suenaga, K., and J.-W. Yoon. 1988. Association of $\beta$-cell-specific expression of endogenous retrovirus with development of insulitis and diabetes in NOD mouse. Diabetes. 37:1722-1726.

8. Charlton, B., A. Bacelj, and T. Mandel. 1988. Administration of silica particles or anti-Lyt 2 antibody prevents $\beta$ cell destruction in NOD mice given cyclophosphamide. Diabetes. 37:930-935.

9. Fujita, H., H. Fujino, K. Nonaka, S. Tarui, and Y. Tochino. 1984. Retrovirus-like particles in pancreatic B-cells of NOD (non-obese diabetic) mice. Biomed. Res. 5:67-70.

10. Fujino-Kurihara, H., H. Fujita, A. Hakura, K. Nonaka, and S. Tarui. 1985. Morphological aspects on pancreatic islets of non-obese diabetic (NOD) mice. Virchows Arch. B Cell Pathol. 49:107-120.

11. Leiter, E. H., and G. L. Wilson. 1988. Viral interactions with pancreatic $\beta$ cells. In The Pathology of the Endocrine Pancreas in Diabetes. P. J. Lefebvre and D. Pipeleers, editors. Springer Verlag, Berlin. 85-105.

12. Appel, M. C., A. A. Rossini, R. M. Williams, and A. A. Like. 1978. Viral studies in streptozotocin-induced pancreatic insulitis. Diabetologia. 15:327-336. 
13. Leiter, E. H., and K. Hamaguchi. 1990. Viruses and diabetes: diabetogenic role for endogenous retroviruses in NOD mice? J. Autoimmun. 3(Suppl):31-40.

14. Leiter, E. H. 1981. The influence of genetic background on the expression of mutations at the diabetes locus in the mouse. IV. Male lethal syndrome in CBA/Lt mice. Diabetes. 30:1035-1044.

15. Leiter, E. H. 1990. The NOD mouse meets the "Nerup Hypothesis". Is diabetogenesis the result of a collection of common alleles present in unfavorable combinations? In Frontiers in Diabetes Research: Lessons from Animal Diabetes III. P. Vardi and E. Shafrir, editors. J. Libby \& Co, London. 54-58.

16. Serreze, D. V., E. H. Leiter, E. L. Kuff, P. Jardieu, and K. Ishizaka. 1988. Molecular mimicry between insulin and retroviral antigen p73. Development of cross-reactive autoantibodies in sera of NOD and C57BL/KsJ- $d b / d b$ mice. Diabetes. 37:351-358.

17. Dickson, C., R. Eisenman, H. Fan, E. Hunter, and N. Teich. 1984. Protein biosynthesis and assembly. In RNA Tumor Viruses: Molecular Biology of Tumor Viruses. R. Weiss, N. Teich, H. Varmus, and J. Coffin, editors. Cold Spring Harbor Laboratory, Cold Spring Harbor, NY. 513-648.

18. Leiter, E. H. 1985. Type C retrovirus production by pancreatic beta cells. Association with accelerated pathogenesis in $\mathrm{C} 3 \mathrm{H}-\mathrm{db} / \mathrm{db}$ ("diabetes") mice. $\mathrm{Am}$. J. Pathol. 119:22-32.

19. Mucenski, M. L., H. G. Bedigian, M. M. Shull, N. G. Copeland, and N. A. Jenkins. 1988. Comparative molecular genetic analysis of lymphomas from six inbred strains. J. Virol. 623:839-846.

20. O’Neill, R. R., A. S. Khan, M. D. Hoggan, J. W. Hartley, M. A. Martin, and R. Repaske. 1986. Specific hybridization probes demonstrate fewer xenotropic than mink cell focus-forming murine leukemia virus env-related sequences in DNAs from inbred laboratory mice. J. Virol. 582:359-366.

21. Chattopadhyay, S., M. Lander, E. Rands, and D. Lowy. 1980. Structure of endogenous murine leukemia virus DNA in mouse genomes. Proc. Natl. Acad. Sci. USA. 77:5774-5778.

22. Stoye, J. P., and J. M. Coffin. 1988. Polymorphism of murine endogenous proviruses revealed by using virus class-specific oligonucleotide probes. $J$. Virol. 62:168-175.

23. Feinberg, A. P., and B. Vogelstein. 1983. A technique for radiolabeling DNA restriction endonuclease fragments to high specific activity. Anal. Biochem 132:6-13.

24. Johnson, K. R. 1991. Improved oligonucleotide labeling and hybridization assay for endogenous nonecotropic murine leukemia proviruses. Mamm. Genome. 1:260-262.

25. Chomczynski, P., and N. Sacchi. 1987. Single-step method of RNA isolation by acid guanidinium thiocyanate-phenol-chloroform extraction. Anal. Biochem. 162:152-159.

26. Hamaguchi, K., and E. H. Leiter. 1990. Comparison of cytokine effects on mouse pancreatic a-cell and $\beta$-cell lines. Viability, secretory function, and MHC antigen expression. Diabetes. 39:415-425.

27. Thurston, S. J., and J. D. Saffer. 1989. Ultraviolet shadowing nucleic acids on nylon membranes. Anal. Biochem. 178:41-42.

28. Frankel, W., J. Stoye, B. Taylor, and J. Coffin. 1990. A linkage map of endogeneous murine leukemia proviruses. Genetics. 124:221-236.

29. Prochazka, M., D. V. Serreze, W. N. Frankel, and E. H. Leiter. 1992. NOR/Lt; MHC-matched diabetes-resistant control strain for NOD mice. Diabetes. 41:98-106.

30. Brilliant, M. H., Y. Gondo, and E. M. Eicher. 1991. Direct molecular identification of the mouse pink-eyed unstable mutation by genome scanning. Science (Wash. DC). 252:566-569.

31. Prochazka, M., D. V. Serreze, S. M. Worthen, and E. H. Leiter. 1989. Genetic control of diabetogenesis in NOD/Lt mice: development and analysis of congenic stocks. Diabetes. 38:1446-1455.

32. Prochazka, M., H. R. Gaskins, L. D. Shultz, and E. H. Leiter. 1992. The NOD-scid mouse: a model for spontaneous thymomagenesis associated with immunodeficiency. Proc. Natl. Acad. Sci. USA. 89:3290-3294.

33. Frankel, W. N., J. P. Stoye, B. A. Taylor, and J. M. Coffin. 1989. Genetic analysis of endogenous xenotropic murine leukemia viruses: association with two common mouse mutations and the viral restriction locus $F v-1$. J. Virol 634:1763-1774.
34. Prochazka, M., H. R. Gaskins, and E. H. Leiter. 1991. Emv-30: a novel ecotropic MuLV present in NOD/Lt and NON/Lt strains. Mouse Genome. $89: 258$

35. Hamaguchi, K., H. R. Gaskins, and E. H. Leiter. 1991. NIT-1, a pancreatic $\beta$ cell line established from a transgenic NOD/Lt mouse. Diabetes. 40:842849.

36. Nakagawa, C., T. Hanafusa, J. Miyagawa, M. Yutsudo, H. Nakajima, K. Yamamoto, N. Kono, A. Hakura, and S. Tarui. 1992. Retrovirus gag protein p30 in the islets of non-obese diabetic mice: relevance for pathogenesis of diabetes. Diabetologia. 35:614-618.

37. Officer, J. E., N. Tecson, J. D. Estes, E. Fontanilla, and R. W. Rongey. 1973. Isolation of a neurotrophic type C virus. Science (Wash. DC). 181:945947.

38. McCarter, J. A., J. K. Ball, and J. V. Frei. 1977. Lower limb paralysis induced in mice by a temperature sensitive mutant of Moloney murine leukemia virus. J. Natl. Cancer Inst. 24:933.

39. Gardner, M. B. 1985. Retroviral spongiform polioencephalomyelopathy. Rev. Infect. Dis. 7:99-103.

40. Murray, A. B., J. Schmidt, and L. Rieke. 1986. Retrovirus-induced osteopetrosis in mice: ultrastructural evidence of early virus production in osteoblasts and osteocytes. Am. J. Pathol. 124:319-323.

41. Harrison, A., and F. Murphy. 1975. Murine oncornavirus activation in the pancreas during infection with Venezuelan equine encephalitis virus. $J$. Natl. Cancer Inst. 55:917-923.

42. Kuff, E. L., and K. K. Lueders. 1988. The intracisternal type A particle gene family: structure and functional aspects. Adv. Cancer Res. 51:183-276.

43. Reuss, F. U., and H. C. Schaller. 1991. cDNA sequence and genomic characterization of intracisternal A-particle-related retroviral elements containing an envelope gene. J. Virol. 65:5702-5709.

44. Merregaert, J., M. Janowski, and E. P. Reddy. 1987. Nucleotide sequence of a radiation leukemia virus. Virology. 158:88-102.

45. Leiter, E., G. Christianson, D. Serreze, A. Ting, and S. Worthen. 1989. MHC antigen induction by interferon $\gamma$ on cultured mouse pancreatic $\beta$ cells and macrophages. Genetic analysis of strain differences and discovery of an "occult" class I-like antigen in NOD/Lt mice. J. Exp. Med. 170:1243-1262.

46. Waters, S. H., J. J. O’Neill, D. T. Melican, and M. C. Appel. 1992. Multiple TCR V $\beta$ usage by infiltrates of young NOD mouse islets of Langerhans: a polymerase chain reaction analysis. Diabetes. 41:308-312.

47. Leiter, E. H. 1989. The genetics of diabetes susceptibility in mice. FASEB (Fed. Am. Soc. Exp. Biol.) J. 3:2231-2241.

48. Pujol-Borrell, R., and G. F. Bottazzo. 1988. Puzzling diabetic transgenic mice. Immunol. Today. 9:303-306.

49. Krieg, A. M., W. C. Gause, M. F. Gourley, and A. F. Steinberg. 1989. A role for endogenous retroviral sequences in the regulation of lymphocyte activation. J. Immunol. 143:2448-2451.

50. Krieg, A. M., and A. D. Steinberg. 1990. Analysis of thymic endogenous retroviral expression in murine lupus. J. Clin. Invest. 86:809-816.

51. Krieg, A. M., M. F. Gourley, and A. D. Steinberg. 1991. Association of murine lupus and thymic full-length endogenous retroviral expression maps to a bone marrow stem cell. J. Immunol. 146:3002-3005.

52. Meruelo, D., A. Rossomando, M. Offer, J. Buxbaum, and A. Pellicer. 1983. Association of endogenous viral loci with genes encoding murine histocompatibility and lymphocyte differentiation antigens. Proc. Natl. Acad. Sci. USA 80:5032-5036.

53. Rossomando, A., and D. Meruelo. 1986. Viral sequences are associated with many histocompatibility genes. Immunogenetics. 23:233-245.

54. Marrack, P., E. Kushnir, and J. Kappler. 1991. A maternally inherited superantigen encoded by a mammary tumor virus. Nature (Lond.). 349:524 526.

55. Frankel, W. N., C. Rudy, J. M. Coffin, and B. T. Huber. 1991. Linkage of Mls genes to endogenous mammary tumor viruses of inbred mice. Nature (Lond.). 349:526-528.

56. Woodland, D. L., M. P. Happ, K. J. Gollob, and E. Palmer. 1991. An endogenous retrovirus mediating deletion of $\alpha \beta \mathrm{T}$ cells? Nature (Lond.)

57. Dyson, P. J. 1991. Genes encoding ligands for deletion of $\mathrm{V} \beta 11 \mathrm{~T}$ cells cosegregate with mammary tumor virus genomes. Nature (Lond.). 349:531-532. 